\title{
An Interference Aware Heuristic Routing Protocol for Wireless Home Automation Networks
}

\author{
Kamanashis Biswas ${ }^{a}$, Vallipuram Muthukkumarasamy ${ }^{a}$, Xin-Wen $\mathrm{Wu}^{a}$, and Kalvinder $\operatorname{Singh}^{a, b}$ \\ ${ }^{a}$ School of Information and Communication Technology, Griffith University, Gold Coast, QLD 4111, Australia \\ Email: kamanashis.biswas@griffithuni.edu.au, \{v.muthu, x.wu\}@griffith.edu.au \\ ${ }^{b}$ IBM Australia Development Laboratory, Gold Coast, QLD 4215, Australia \\ Email: kalsingh@au.ibm.com
}

\begin{abstract}
Wireless Home Automation Networks consist of battery powered sensors and actuators that communicate with each other over wireless channels. The sensor nodes collect environmental information such as temperature, light intensity, humidity, and pressure to provide context-aware services and to facilitate smart home control. However, resource limitations, unpredictable topology changes, and interference introduce a number of challenges to the design of home automation protocols. One of the challenging tasks is developing energy efficient routing protocols to prolong the network lifetime. This paper proposes an interference aware heuristic routing protocol which determines the forwarding path between sensors and the base station using a heuristic function and a heuristic search algorithm. On the basis of local and global distance, longevity factor (LF), and link quality (LQ), the heuristic function generates a heuristic value for each node and this value is used in $A^{*}$ search algorithm to determine the efficient route. Simulation results show that the proposed routing scheme outperforms GAHR and $A O D V_{j r}$ protocols in terms of network lifetime, energy consumption, and message throughput. Furthermore, it achieves up to $15 \%$ increase in packet delivery ratio over GAHR protocol in a noisy environment.
\end{abstract}

Index Terms $-A^{*}$ search, heuristic function, home automation networks, interference, link quality, longevity factor

\section{INTRODUCTION}

$\mathbf{R}$ APID advances in microsensor technology and wireless communications have expanded the application domains of wireless sensor networks (WSNs) including industry control, structural health monitoring, strategic surveillance, and wireless home automation. Among the wide variety of WSN applications, wireless home automation networks (WHANs) have gained considerable attention both in academia and industry over the past few years. Moreover, recent research indicates that it will be one of the fastest growth sectors in consumer electronics over the next five years [1].

A WHAN consists of a number of sensors and actuators that sense and forward collected information to the base station in order to monitor and control devices in a home network. As depicted in Figure 1, the WHAN contains a gateway server (GS), a base station (BS), and sensor nodes (SNs). The SNs are spread over the house to collect various data and send them to the $\mathrm{BS}$ via other nodes within one hop distance. The BS is responsible for the

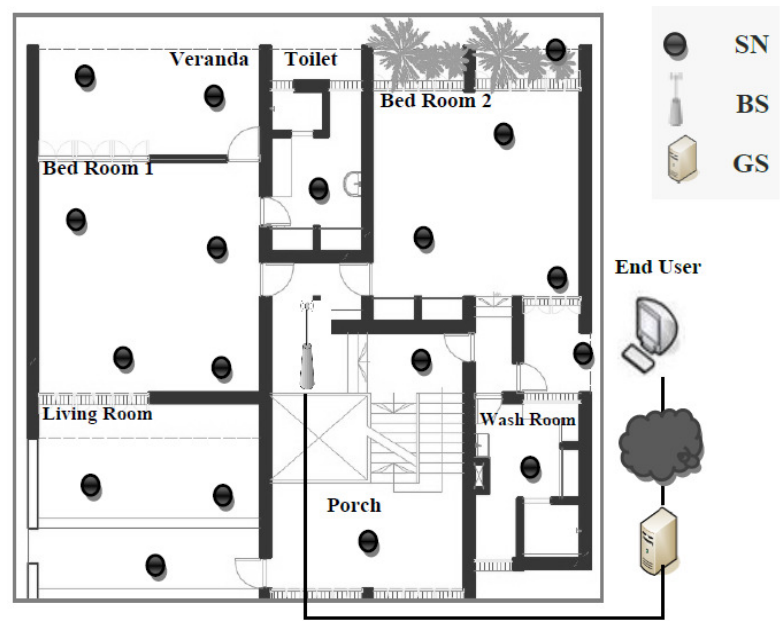

Figure 1. An example of wireless home automation network

maintenance of entire network, whereas the GS connects the BS with end user in order to provide external controls. Compared to battery operated SNs, the BS has more computation, memory, and energy resources. Although battery powered SNs are easy to install, they need to be replaced or recharged on a regular basis. Statistics show that in about $50 \%$ of in-home displays (IHD), batteries do not get replaced when they run out of power [2]. Therefore, energy efficient routing protocol is essential to improve the lifetime of WHANs.

Many routing protocols have been proposed for WHANs to extend network lifetime [3], [4]. These protocols reduce total energy consumption by sacrificing uniform energy use in the network and thus result in quick network partitions. In addition, WHAN is subject to interference due to presence of numerous devices and networks in home environment such as WiFi, Bluetooth, smart meter, cordless phones, and microwave ovens [5]. However, very few protocols consider interference as one of the important parameters in a WHAN routing protocol design in contrast to routing protocols in wireless sensor networks.

This paper proposes an interference aware heuristic routing (IAHR) scheme to determine the efficient route 
between a $\mathrm{SN}$ and the BS. The main contributions of this paper are two-fold. First we present IAHR, a heuristic routing protocol based on both local and global knowledge that significantly improves the network performance. Furthermore, instead of considering interference as a binary event (i.e., yes or no), IAHR protocol quantizes the level of interference of each link and uses the value as one of the routing parameters. Thus, the protocol takes every opportunity of delivering data packets using low-interference links and keeps the network functional. Finally, we investigate the effects of random changes in topology and compare performance of the proposed algorithm with standard specifications.

The rest of the paper is organized as follows. Section II describes related works. Section III details the network architecture, and routing procedure of the proposed protocol with three different scenarios. Section IV presents experimental results and performance analysis of the proposed protocol. Finally, Section V concludes the paper.

\section{RELATED WORKS}

A number of standard solutions have been proposed for WHANs such as ZigBee, Z-Wave, INSTEON, Wavenis, Bluetooth, WiFi, and UWB (Ultra-Wideband) [6], [7]. The ZigBee model enables multihop data delivery, where the routes are created by using either Ad hoc On-demand Distance Vector (AODV) or simplified AODV (AODV $\left.\mathrm{jr}_{\mathrm{jr}}\right)$ routing protocol for point-to-point communication [8][10]. On the other hand, the ZigBee PRO solution facilitates many-to-one communication and thus is more suitable for WHANs. In Z-Wave, a controller maintains the entire network topology and determines the routes for every SN [11]. When a packet is required to transmit, the controller includes the path to reach the destination node. Unlike ZigBee and Z-Wave, INSTEON uses a different multihop routing approach (simulcast), where SNs within the same radio range are allowed to transmit messages simultaneously [12]. Wavenis [13] is a wireless protocol stack that constructs a four-level virtual hierarchical tree. The root node works as the BS or gateway and gathers data packets from lower level nodes. Compared to these technologies, Bluetooth and UWB support very low communication range and limited number of nodes, therefore, are not suitable for large-scale WHANs [7]. Similarly, WiFi is not feasible for WHANs due to high energy consumption.

One of the drawbacks of these protocols is that very few of them consider link quality as a routing metric. For example, Z-Wave, INSTEON, Bluetooth, and UWB are susceptible to interference and is not suitable for the applications communicating over noisy channel. Similarly, Wavenis uses Received Signal Strength Indicator (RSSI) value to estimate link quality which may be inaccurate because of multipath and interference [6]. Another drawback is that most of these WHAN architectures (ZigBee, INSTEON, and UWB) implement flooding-based routing protocols that are subject to high energy consumption and high probability of collision in the MAC (media access control) layer.

Relative Direction based Sensor Routing (RDSR) [3] divides the entire home area into a number of sectors and assigns a manager node to each sector. The manager nodes receive data from the $\mathrm{SNs}$ and send them to the BS. However, node energy and link quality are not considered in this protocol. As a result, it may not find the efficient route and may result in high energy consumption and high delay. Furthermore, RDSR protocol does not support node mobility.

Base-station Centric Data Gathering Protocol (BCDGP) [4] is a cluster-based hierarchical routing algorithm proposed for home automation applications. The protocol uses relay nodes for each room of the house to receive information from sensor nodes. However, cluster-based approach is not suitable for WHANs since it consumes significant amount of energy to build and maintain efficient cluster structure. In addition, link quality is not considered in this protocol.

A Location-Based self-Adaptive Routing (LBAR) protocol is proposed to extend network lifetime in WHANs [14]. The protocol limits route discovery flooding to a cylindrical request zone. Experimental results show that LBAR protocol performs better than $A_{0 D V}$ in terms of routing overhead and packet delivery ratio. However, the protocol is not energy efficient and is not suitable for a noisy channel.

A Greedy and $A^{*}$ Heuristic Routing (GAHR) algorithm based on Euclidean distance is proposed for WHANs [15]. The algorithm uses greedy forwarding to limit the number of hops required for data transmission. Furthermore, the protocol implements $A^{*}$ search algorithm to overcome unpredictable topology changes and local minimum problems. In GAHR, every SN maintains a neighbor table that keeps a record of interfered links. When a SN detects data transmission error, it marks the forwarding node as temporarily unstable and sets the timer. The temporarily unstable node waits to return to stable state until the timer expires. The number of recoveries from temporarily unstable state to stable state is also recorded. When the recovery count is greater than a threshold value, the corresponding link is marked unstable. This protocol has a number of limitations. First, since the node energy is not used in route selection procedure, low energy SNs can be selected as forwarding nodes and may run out of energy fast. Second, the greedy forwarding selects the same route until a node on that path exhausts its energy. Thus, the protocol results in network partitions. Third, if a $\mathrm{SN}$ is marked unstable then it will never return to stable state. For example, a microwave oven is a source of radio interference in home network. In an office, the oven is used several times to heat or cook food during office hours. Hence, the SNs near a microwave oven will be affected many times and hence may be marked as unstable. It is more likely that there will be no interference at night or on a public holiday. Therefore, GAHR protocol is unable to deal with interference problems effectively. 
Our previous work on Energy Aware Heuristic-based Routing (EAHR) protocol [16] proposed a simple heuristic function to find the efficient route between a SN and the BS. The protocol determines optimal path between source and destination nodes using local and global distance when the nodes have a sufficient amount of energy. If the energy level goes below the threshold limit then it uses energy as a routing parameter to find alternative path to reach the BS. However, the protocol has few limitations. First, it does not consider link quality although performance of home automation network is highly affected by interference. Second, no experiment is done to evaluate the impacts of node mobility in the network. Since a home network also contains mobile devices, the effectiveness of the protocol is unknown. Finally, EAHR takes energy level in consideration when nodes are already in critical conditions. Thus, the protocol is unable to take full advantages of energy balanced routing. To overcome these problems, we have proposed a new interference aware heuristic routing protocol with improved adaptability to changes in topology. Furthermore, we have conducted extensive experiments to evaluate the effectiveness and performance of IAHR protocol in home environments.

\section{InTERfEREnCE AwARE Heuristic Routing PROTOCOL}

This section presents the network model, radio model, and the proposed IAHR protocol in details.

\section{A. Network Model}

It is assumed that WHAN comprises of a number of SNs, a BS, and a GS. Further, the following assumptions are considered to define the network model.

- Every SN has a unique node ID and the node is aware of the location of the BS as well as its own. The location of the static nodes can be determined during network deployment using either physical devices (e.g., Global Positioning System) or topology discovery algorithms [17] whereas the BS broadcasts its location to all SNs.

- SNs periodically send remaining energy and link quality values to their neighbor nodes. To adapt to the changes in topology caused by intermittent interference and energy consumption, every SN collects local information from its neighbour nodes at the beginning of each round.

- SNs are equipped with limited batteries, whereas the BS has sufficiently large power. Since SNs are operated by battery power, they have limited energy. On the other hand, the BS is supported with powerful resources and extra capabilities as it connects a WHAN to a wired network.

- The network consists of both stationary and mobile nodes and the energy consumption is not uniform for every SN.

\section{B. Radio Model}

The energy consumption model for radio communication is assumed similar to Heinzelman et al. radio model [18]. We have used the multipath propagation model which is based on the distance between the transmitter and the receiver. In order to receive $k$-bit message and transmit $k$-bit message over the distance $d$, the radio hardware dissipates energy as per following equations respectively.

$$
\begin{gathered}
E_{R x}(k)=k \epsilon_{\text {elec }} \\
E_{T x}(k, d)=k \epsilon_{\text {elec }}+k \epsilon_{\text {amp }} d^{\gamma}
\end{gathered}
$$

where, $\epsilon_{\text {elec }}$ and $\epsilon_{a m p}$ denote electronics energy and amplifier energy respectively, and $\gamma$ represents the pathloss exponent. The value of $\gamma$ is set to 3 , which is typical for home and office environments [19].

\section{The IAHR Protocol}

WHAN may have both static and mobile nodes. Every SN exchanges the longevity factor and location information with its neighbor nodes during the network setup phase. However, the network is considered interference free at this stage and thus the $L Q$ value is set to zero for all SNs initially. In order to adapt to the changes in the network topology, the SNs share the $L F$ and $L Q$ value at the beginning of every round with their neighbor nodes. Since WHAN is a small network, the overhead for exchanging local information is reasonable.

Node mobility is another critical issue in WHANs. It is quite likely that the number of mobile nodes is smaller compared to the static nodes in home network. In IAHR protocol, the static nodes keep separate records of the mobile nodes and they usually do not select mobile nodes to route data packets. When a mobile node has to transmit data packets, it sends a 'Hello' message to discover the neighbor nodes within its communication range. Upon receiving the reply messages with location information, the mobile node selects the neighbor node with closest distance to forward data packets. However, static nodes may use mobile nodes if and only if there is no route available to reach the BS through static sensors. Thus, IAHR protocol makes every effort to keep the network functional.

1) Heuristic Function: The heuristic function guides the search procedure by providing a good estimate of how far the destination node is from the current node. It derives a numerical value for every $\mathrm{SN}$ by combining different routing parameters. The following parameters are used in the heuristic function of IAHR protocol.

a) Local and Global Distance- The local and global information on network topology helps to find an efficient route. Since the use of only local or global information may lead to network partition and local minimum problems, IAHR protocol uses the distance traversed so far (i.e., local distance) as well as estimated distance between a SN and the BS (i.e., global distance) to guide the search procedure in the right direction. 


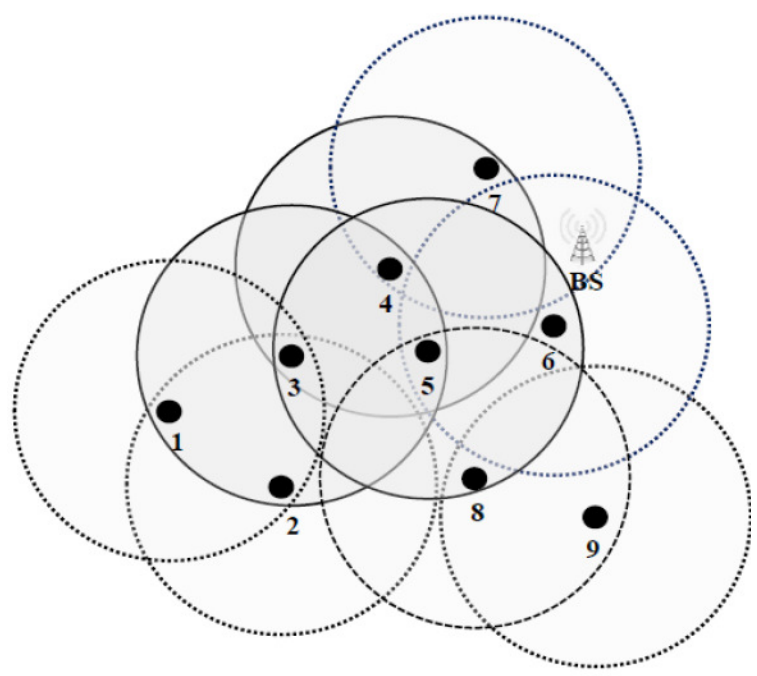

Figure 2. Impacts of greedy forwarding technique

b) Longevity Factor- Remaining energy is an important routing metric that can help to extend network lifetime by avoiding low energy nodes during route selection process. The IAHR protocol derives longevity factor $(L F)$ for every $\mathrm{SN}$ from remaining energy as per following equation:

$$
L F=\frac{T \times E_{\text {res }}}{E_{\text {init }}}
$$

where, $T$ is sustainable time of a $\mathrm{SN}, E_{\text {res }}$ and $E_{\text {init }}$ are remaining energy and initial energy of that $\mathrm{SN}$ respectively. The $L F$ metric is used to maximize the value of the heuristic function to avoid low energy nodes from being selected repeatedly.

Figure 2 presents a network topology with nine SNs and a BS. Let the SNs send information to the BS using greedy forwarding algorithm proposed in GAHR protocol. The greedy forwarding technique uses global distance between SNs and the BS to select the forwarding nodes. Suppose, the global distance between node 4 and the BS is 25 , whereas the distance is 22 for node 5 . Hence, the packets from node 1-3, 8, and 9 will go through node 5 in this method. As a result, node 5 will deplete its energy and node 8 and node 9 will be disconnected from the network. Even the use of $A^{*}$ search algorithm fails to overcome the situation if the route 'node 5 -node $6-B S$ ' is better than the route 'node $4-$ node $7-B S$ '. The proposed protocol overcomes this problem by using longevity factor in addition to local and global distance. Since the decrease in the energy level of a node increases the heuristic value of that node, the probability of selecting alternative nodes is very high in IAHR protocol. For example, the heuristic value of node 5 will be increased with the decrease in remaining energy. Therefore, the packets from node 1 3 will go through node 4 instead of node 5 when the heuristic value of node 4 is smaller than that of node 5 .

c) $L Q$ Metric- Link quality in WHANs is highly affected by interference. Interference leads to packet retransmissions and thus wastes significant amount of energy. However, the existing protocols always consider interference as a binary event. But, the real fact is that it has a continuous degree of impact on reception of packets depending on Signal-to-Interference-and-Noise Ratio (SINR) [20].

Most of the network models assume that a packet is received correctly if the SINR value is above the threshold level. However, research indicates that the SINR threshold is not a constant value and it mainly depends on transmitter hardware and signal strength [21]. For example, experimental results on Mica2 sensor mote find the following SINR thresholds for successful packet reception: $\mathrm{SINR}_{\mathrm{LOW}}=2 \mathrm{~dB} ; \mathrm{SINR}_{\mathrm{HIGH}}=6 \mathrm{~dB}$. The $\mathrm{SINR}_{\mathrm{LOW}}$ threshold level indicates that if the SINR is equal to or less than $2 \mathrm{~dB}$ then the packet cannot be decoded. On the other hand, if the SINR value is equal to or greater than $6 \mathrm{~dB}$, the packet will be received successfully. It is noted that the probability of successful packet reception increases with the increase of the SINR value. As an example, if the $S I N R$ value is $5 \mathrm{~dB}$ then there is more than $80 \%$ probability that a packet will be decoded correctly, whereas the probability is about $10 \%$ for $4 \mathrm{~dB}$ SINR [21]. IAHR protocol takes the advantage of this SINR characteristic and quantifies SINR level in such a way that the value can directly be used in route selection procedure.

In IAHR protocol, the receiver node calculates the level of interference using the geometric SINR model [22] at each round. For example, if node $v$ sends data packets to node $u$, then node $u$ measures the SINR of link ' $u$-v' as follows:

$$
S I N R(u v)=\frac{S_{v u}}{I_{v u}+N}
$$

where, $S_{v u}$ denotes the signal strength of sender $v, I_{v u}$ is the total signal power received from other nodes except $v$ and $N$ is noise power. The value of $S_{v u}$ is the product of path gain $\left(G_{v u}\right)$ and power $\left(P_{v}\right)$ whereas $I_{v u}$ is the sum of the signal power of other nodes, i.e., $I_{v u}=\Sigma G_{w u} P_{w}$, for $w \neq v$. On the basis of SINR value, each node is assigned a numerical value which presents the quality of link between the sender and the receiver. For example, if $S I N R \geq 6 \mathrm{~dB}$, the link is not affected at all. In contrast, the link is completely down when $\operatorname{SINR} \leq 2 \mathrm{~dB}$. Thus, the effect of interference and noise on a link $(u-v)$ is defined as per following equation:

$$
L Q(u-v)= \begin{cases}0 & ; \operatorname{SINR}(u v) \geq 6 \\ 100 & ; \operatorname{SINR}(u v) \leq 2 \\ \left(S I N R_{H I G H}-S I N R_{u v}\right)^{2} & ; \text { otherwise }\end{cases}
$$

The $L Q$ value is set to 100 if the SINR is equal to or less than the lower threshold. This is done to increase the heuristic value of the interfered links so that the link becomes unavailable for the current round. Similarly, the closer the SINR value is to the upper threshold, the less the $L Q$ value is used to represent it. As an example, if the $S I N R$ of a link is $5 \mathrm{~dB}$, then the $L Q$ value will be $(6-5)^{2}$, i.e., 1. Thus, the proposed protocol represents the interference using different weights on the basis of link quality. Now, combining the four parameters, node $v$ 


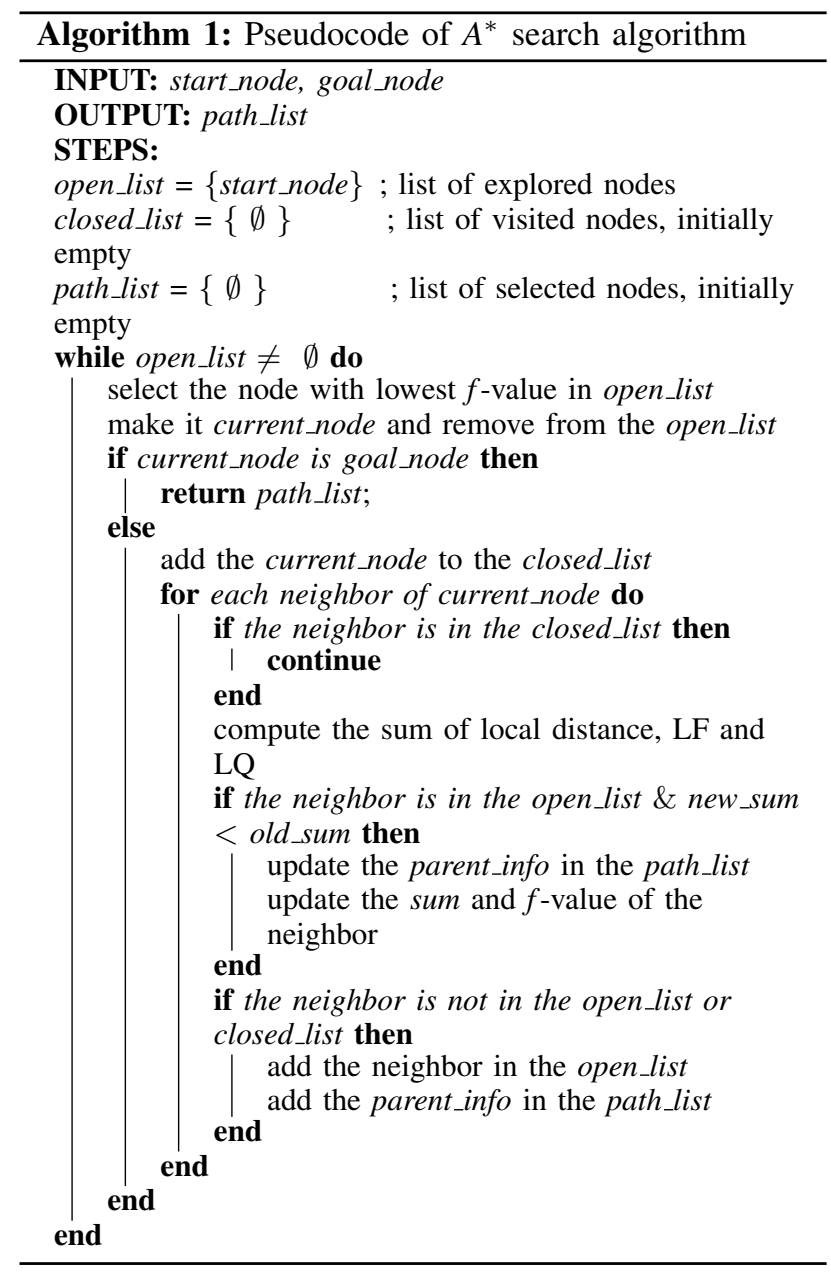

computes the heuristic value of node $u$ as follows:

$$
f_{v}(u)=g(u)+h(u)+100 / L F(u)+L Q(u-v)
$$

where, $f_{v}(u)$ is the heuristic value of node $u, g(u)$ and $h(u)$ are local and global distance respectively, $L F(u)$ is the longevity factor, and $L Q(u-v)$ is the quality of link between node $u$ and node $v$. The inverse of $L F$ is multiplied by 100 to increase the heuristic value of low energy nodes in the equation.

2) $A^{*}$ Search Algorithm: $A^{*}$ search combines bestfirst-search and Dijkstra's algorithm to find the efficient route between source and destination nodes. This is a leading path-finding algorithm used in real-world applications such as the Warcraft III game, and road networks [23]. $A^{*}$ search is more efficient than other conventional shortest path algorithms such as Bellman-Ford and Dijkstra's algorithms. The conventional search algorithms are not applicable in WHANs due to long delay, trapping in blind alley, and computational complexity. Moreover, it is difficult for them to adapt with frequent changes in network topology. However, $A^{*}$ search overcomes these problems effectively. To efficiently compute the optimal solution, it combines features of both pure heuristic search and uniform cost search.

$A^{*}$ search algorithm maintains an open_list and a closed_list as shown in Algorithm 1, where the open_list initially contains the start_node and the closed_list is empty. Then it removes the first node from the open_list and makes it the current_node. After that, the algorithm explores all neighbors of the current_node, computes their heuristic values, and adds them to the open_list. The open_list is then sorted in ascending order with respect to heuristic value and the current_node is added to the closed_list as well as in the path_list. The path_list is an array that keeps track of the valid path by updating the traversed nodes accordingly. Now, it again removes the first node from the open_list, makes it the current_node, and follows the same procedure until the goal state is found. In a nutshell, $A^{*}$ search follows a path of the lowest known heuristic cost and also maintains a priority queue of alternative path segments along the way. Thus, it is not trapped in an infinite loop and reduces delay, and complexity. Since WHAN consists of resourceconstrained SNs, $A^{*}$ heuristic search algorithm is more suitable compared to conventional algorithms.

3) Routing Procedure: As mentioned earlier, the heuristic function of the proposed IAHR protocol uses four parameters to calculate the heuristic value $f$ of a node: the distance traversed to reach current node from source node $(g)$, the Euclidian distance between current node and the BS $(h)$, the longevity factor $(L F)$, and the link quality $(L Q)$. As an example, Figure 3 depicts a network topology with $15 \mathrm{SNs}$ and a BS. The communication range of the $\mathrm{SNs}$ is presented by dotted circles, whereas the dotted lines represent the communication links between two nodes. Every node computes the route to reach the $\mathrm{BS}$ using $A^{*}$ search algorithm. In order to understand the impacts of remaining energy and interference in WHANs, three different cases are explained for the proposed protocol.

The first case assumes that all SNs have same level

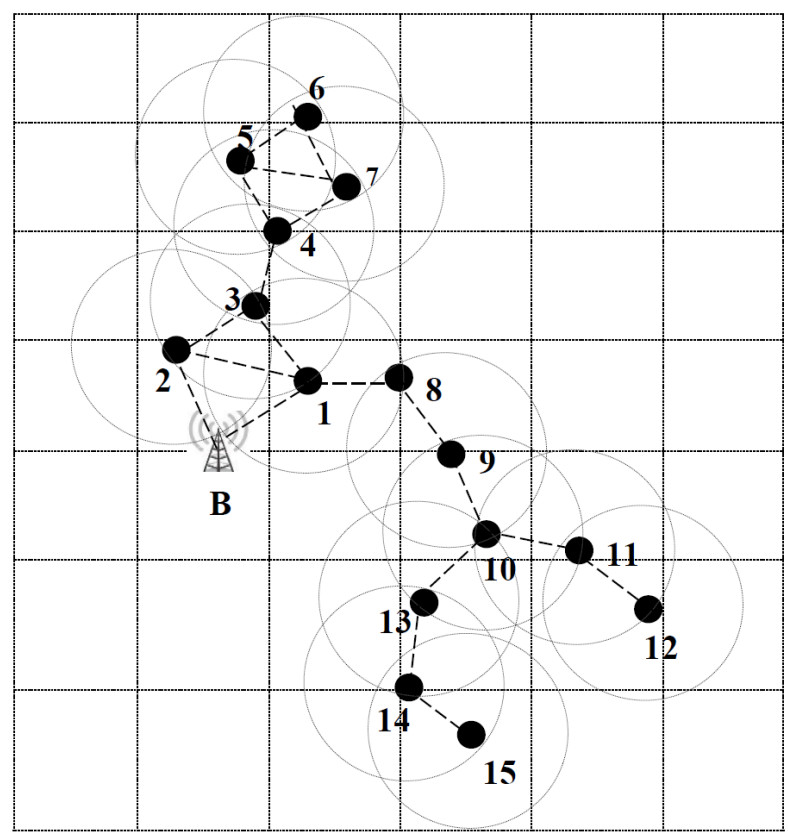

Figure 3. An example of WHAN topology 
of energy and there is no interference in the network. Suppose, node 6 has to find the route to reach the BS and the initial parameters of its neighbor nodes are as follows: $[g(5)=1, g(7)=3] ;[h(5)=10, h(7)=11]$; $[\operatorname{eng}(5)=\operatorname{eng}(7)=0.5 \mathrm{~J}] ;[L Q(5-6)=L Q(7-6)=0] ; T$ $=50$ minutes for both nodes. First, node 6 computes its $f$-value via node 5 and node 7 as follows: $f(5)=1+10$ $+2+0=13, f(7)=3+11+2+0=16$. Since the $f$-value via node 5 is smaller than that of node 7 , node 6 selects node 5 as its forwarding node. Upon receiving the information, all the neighbor nodes update their $f$-values with respect to node 6. During the next hop selection process, node 5 calculates the $f$-value to reach the BS via node 7 and node 4 . Let, the values are: $f(4)=11 ; f(7)$ $=15$ respectively. Hence, node 4 is selected as the next hop as well as the $f$-value and $g$-value of node 7 is also updated. In this way, the route of node 6 is constructed to reach the $\mathrm{BS}$.

The second case assumes that the SNs have different level of energy and the network is still interference free. Suppose, after time $t$, the energy level of node 5 and node 7 degrades to $0.1 \mathrm{~J}$ and $0.25 \mathrm{~J}$ respectively. At this stage, the new $f$-values of node 5 and node 7 are: $f(5)=1+10$ $+100 /((0.150) / 0.5)+0=21$ and $f(7)=3+11+100 /$ $((0.2550) / 0.5)+0=18$ respectively. Since the heuristic value of node 7 is smaller than that of node 5 , node 7 will be selected to forward the data packets of node 6 . It means that IAHR protocol avoids low energy nodes when there exists alternative routes. Thus, the protocol ensures energy balanced routing in WHANs.

The third case explains the effects of both remaining energy and interference. Consider, the following parameters of node 5 and node 7: $[\operatorname{eng}(5)=0.2$, eng $(7)=0.5]$; $[L Q(5-6)=0, L Q(7-6)=4]$. Using the values, node 6 computes the heuristic values as follows: $f(5)=1+10+$ $100 /((0.250) / 0.5)+0=16$ and $f(7)=3+11+100 /((0.5$ $50) / 0.5)+4=20$. Although node 7 possesses more energy than node 5, it will not be allowed to forward data packets. It indicates that IAHR protocol effectively handles the interference problem and minimizes the number of packet retransmissions.

\section{EXPERIMENTS AND ANALYSIS}

The proposed protocol is implemented in the OM$\mathrm{NET}++$ simulator and compared with $\mathrm{AODV}_{\mathrm{jr}}$ and GAHR protocols. AODV $\mathrm{jr}$ is a widely used standard for WHANs, whereas GAHR uses similar routing parameters like IAHR protocol. It incorporates interference in addition to node mobility, local and global distance. Five performance metrics are chosen to evaluate and compare the efficiency and reliability of IAHR, GAHR, and $\mathrm{AODV}_{\mathrm{jr}}$ protocols. They are Packet Delivery Ratio (PDR), Remaining Energy, Network Lifetime, Routing Overhead, and Average Packet Delay. The simulation parameters used in the experiments are listed in Table I.
TABLE I.

SIMULATION PARAMETERS

\begin{tabular}{ll}
\hline Parameters & Value \\
\hline Network Grid & $(0,0)$ to $(100,100)$ \\
Location of BS & $(50,50)$ \\
Initial Energy & $2 \mathrm{~J}$ \\
Transmission Range & $20 \mathrm{~m}$ \\
Data Packet Size & $128 \mathrm{bytes}$ \\
Broadcast Packet Size & $24 \mathrm{bytes}$ \\
$\epsilon_{\text {elec }}$ & $50 \mathrm{~nJ} / \mathrm{bit}$ \\
$\epsilon_{\text {amp }}$ & $10 \mathrm{pJ} / \mathrm{bit} / \mathrm{m}^{2}$ \\
$\gamma$ & $3(\mathrm{lossy} \mathrm{medium})$ \\
\hline
\end{tabular}

\section{A. Simulation Scenarios}

Two different scenarios are designed to evaluate the performance of the proposed protocol. The number of mobile nodes in both scenarios is two, whereas the number of interferer nodes is two in the first scenario and three in the second scenario. To observe the impacts of increased network connectivity, the experiments are performed for 50, 60, and 70 nodes respectively. Furthermore, the simulation is performed for 500 times for every experiment to compute the average value.

\section{B. Simulation Results and Analysis}

1) Performance comparison: The Scenario 1 is designed to investigate the message throughput, network lifetime, and total energy consumption in IAHR, GAHR, and $\mathrm{AODV}_{\mathrm{jr}}$ protocols. This scenario uses two mobile nodes and two interferer nodes. The speed of both mobile nodes is set to 0.5 meter/second. One interferer node generates random level of interference by varying its transmission power from $-10 \mathrm{~dB}$ to $2 \mathrm{~dB}$ whereas the transmission power of the other node is fixed to $-10 \mathrm{~dB}$.

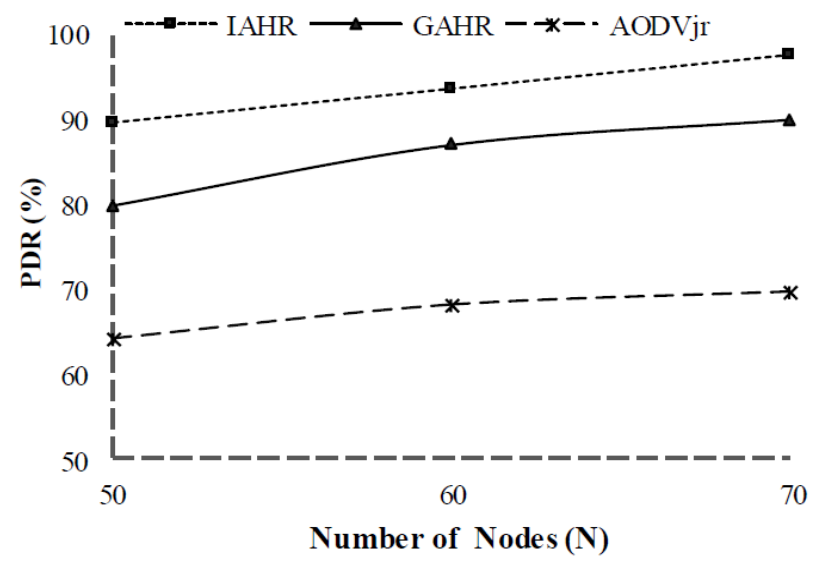

Figure 4. Comparison of packet delivery ratio (Scenario 1)

Figure 4 shows the packet delivery ratios for different number of sensor nodes. It can be seen that IAHR protocol achieves higher level of PDR than the other two protocols. GAHR protocol mainly uses greedy search algorithm to construct the routes. Since greedy forwarding results in quick network partition, many SNs are disconnected 


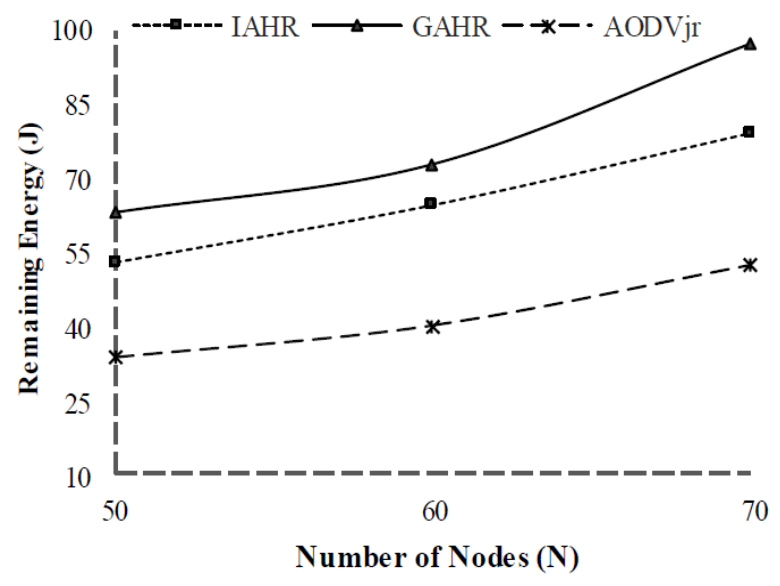

Figure 5. Comparison of remaining energy

and unable to reach the BS. This does not happen in IAHR protocol since it maintains a balance in energy consumption of the SNs. The heuristic function of IAHR protocol maximizes the heuristic values of energy critical nodes and thus finds alternative route to send data packets. On the other hand, the AODV $\mathrm{jr}$ protocol floods the route request packets to the entire network that leads to heavy contention and collisions in the MAC layer. The SNs in $\mathrm{AODV}_{\mathrm{jr}}$ deplete their energy quickly and partition the network. The graph also indicates that the PDR is increased with the increase of SNs. Since the network area is fixed, the growth in node density increases network connectivity as well as the total number of delivered packets.

Figure 5 shows the amount of remaining energy in IAHR, GAHR, and AODV jr protocols until the network becomes non-functional. The network is considered nonfunctional when $10 \%$ of the total nodes run out of power. It can be seen that the amount of remaining energy is maximum in GAHR protocol. This remaining energy is wasted since at that time the network is down and unable to continue its operation. GAHR protocol does not consider node energy to compute forwarding path. Hence, the protocol may use critical nodes to transmit data packets until they exhaust their energy. Therefore, the nodes dependent on critical nodes to send information to the BS are quickly disconnected from the network although they have sufficient remaining energy. The graph also shows that the amount of remaining energy in $\mathrm{AODV}_{\mathrm{jr}}$ is lowest among all. However, this result does not indicate the energy efficiency of $\mathrm{AODV}_{\mathrm{jr}}$ protocol. The reason is that $\mathrm{SNs}$ in $\mathrm{AODV}_{\mathrm{jr}}$ waste significant amount of energy because of flood-based route discovery mechanism. The frequent packet collisions and retransmissions are mainly responsible for energy drainage in $\mathrm{AODV}_{\mathrm{jr}}$ protocol. Therefore, it is obvious that IAHR protocol makes better use of node energy than the other two protocols. Since IAHR protocol implements energy balanced routing, nodes remain alive for more rounds and consumes more energy due to increased volume of data transmissions compared to GAHR and AODV $\mathrm{jr}$ protocols.

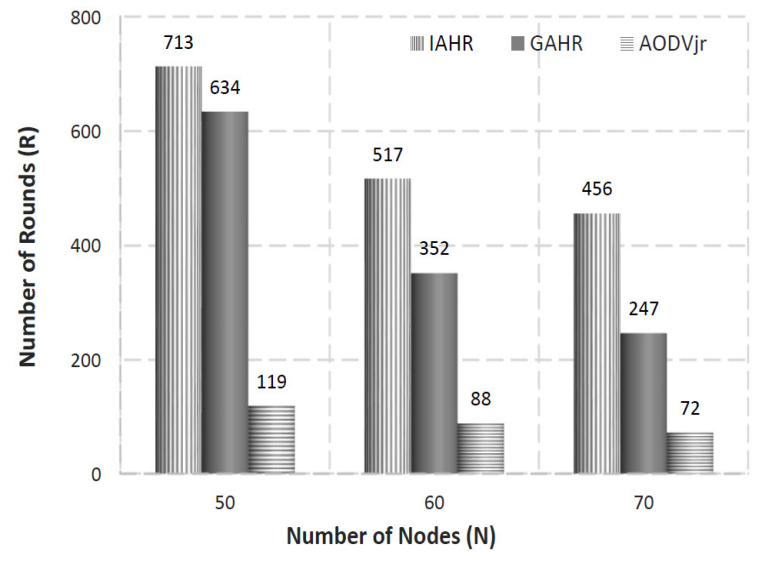

Figure 6. Network lifetime comparison

The simulation result on network lifetime comparison of IAHR, GAHR, and $\mathrm{AODV}_{\mathrm{jr}}$ protocols is illustrated in Figure 6. The results indicate that the network (with 50 nodes) remains functional for 713 rounds in IAHR protocol, whereas the number is 634 and 119 for GAHR and $A O D V_{j r}$ protocols respectively. The similar trend is observed for networks consisting of 60 and 70 nodes respectively. Furthermore, the graph shows that the network lifetime decreases with the increase of number of nodes in the network. The reason is that the increased number of nodes results in increased volume of data traffic, which leads to a high probability of collisions in the network. Even in this situation, IAHR protocol outperforms the other two protocols. Every node in IAHR protocol is aware of link quality and remaining energy of its 1-hop neighbours at the beginning of each round. This local information helps the protocol to avoid unsuccessful data transmission through high-interference links. Furthermore, exclusion of low energy nodes in route selection process delays network partitions. Therefore, nodes remain alive for more rounds in IAHR than the other two protocols.

2) Performance comparison in presence of high interference: In Scenario 2, the experiments are performed to measure the PDR, routing overhead and average packet delay. The $\mathrm{AODV}_{\mathrm{jr}}$ protocol is not evaluated since it does not use link quality in route selection procedure. However, this scenario uses three interferer nodes where two nodes have the same properties defined in Scenario 1. The third node is mobile and generates random level of interference.

Figure 7 indicates that IAHR protocol achieves 10$15 \%$ more PDR gain compared to GAHR protocol in noisy communication environment. In IAHR, every SN is aware of the link quality of its neighbors and can easily select alternative path to avoid the interfered links. If the interfered links are recovered then the information is provided to the corresponding node at the beginning of new round. Therefore, the links can again be used in forwarding data packets. On the other hand, GAHR protocol marks a link permanently unstable if it exceeds the threshold level of recovery count. This approach 


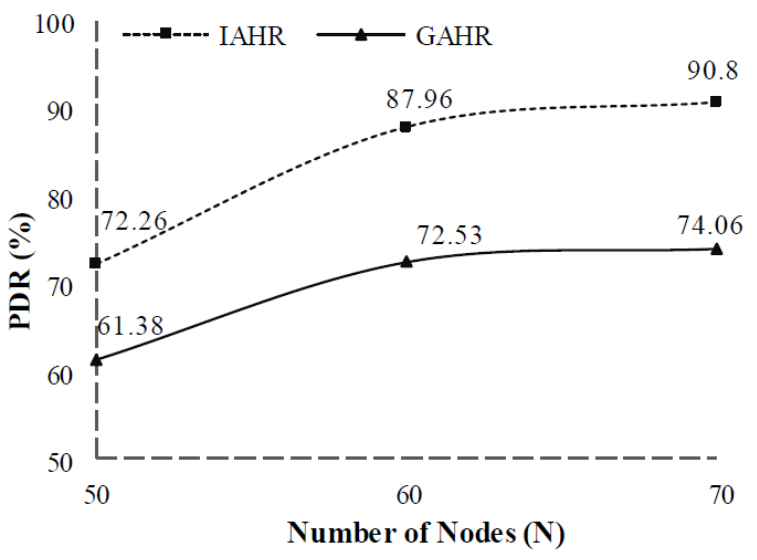

Figure 7. Comparison of packet delivery ratio (Scenario 2)

reduces node connectivity and thus many packets are unable to reach the BS.

Table II represents routing overhead and average packet delay of IAHR and GAHR protocols. It can be seen that GAHR protocol sends more control packets compared to IAHR protocol. GAHR protocol follows greedy forwarding technique and restarts the route discovery process using $A^{*}$ search algorithm to overcome the local minimum problem. Furthermore, since the protocol detects the interference in real time, it has to find new routes to avoid interfered links. Therefore, GAHR protocol has to send more control packets than IAHR protocol. For the same reason, the average packet delay in GAHR is also higher than that of IAHR protocol. Instead of marking a node unstable, IAHR protocol quantizes the level of interference in order to use the low interfered links for data transmission. In the presence of mobile interferers, this strategy significantly minimizes delay since the affected links become functional as soon as they recover from the interference.

TABLE II.

COMPARISON ON ROUTING OVERHEAD AND LATENCY

\begin{tabular}{llll}
\hline Protocols & $\begin{array}{l}\text { Total } \\
\text { Nodes }\end{array}$ & $\begin{array}{l}\text { Routing } \\
\text { Overhead }\end{array}$ & $\begin{array}{l}\text { Avg. Packet } \\
\text { Delay (s) }\end{array}$ \\
\hline \multirow{4}{*}{ IAHR } & 50 & 1611 & 0.0207 \\
& 60 & 1783 & 0.0236 \\
& 70 & 2276 & 0.0254 \\
\hline \multirow{4}{*}{ GAHR } & 50 & 1634 & 0.0317 \\
& 60 & 1857 & 0.0343 \\
& 70 & 2388 & 0.0392 \\
\hline
\end{tabular}

\section{CONCLUSION}

This paper proposed an interference aware heuristic routing protocol for WHANs to extend network lifetime. The proposed scheme uses $A^{*}$ search algorithm to find an efficient route on the basis of a number of routing parameters. In IAHR protocol, every $\mathrm{SN}$ is aware of local and global distance, remaining energy, and link quality of its neighbor nodes before starting the route selection process. Therefore, the low energy nodes and high-interference links are avoided when selecting the routes. Thus, the network lifetime improves over that of GAHR and $\mathrm{AODV}_{\mathrm{jr}}$ protocols by a factor upto 1.84 and 6.36 respectively. Similarly, the throughput of IAHR protocol is increased by maximum of $10 \%$ and $25 \%$ over GAHR and AODV jr protocols respectively. Although IAHR protocol is designed for WHANs, it can be used in large-scale WSN applications because of its selfadaptation characteristic. The future work will focus on a real world implementation of the protocol in a home environment.

\section{REFERENCES}

[1] Transparency Market Research, "Home automation market (lighting, safety and security, entertainment, HVAC, energy management) - global industry analysis, size, share, growth, trends, and forecast, 2013 - 2019," Market research report, pp. 1-122, Oct. 2013.

[2] Sentec, "Innovation challenges in the home automation market," White paper, pp. 1-5, 2014.

[3] H. Oh, H. Bahn, and K. Chae, "An energy-efficient sensor routing scheme for home automation networks," IEEE Trans. of Cons. Elec., vol. 51, no. 3, pp. 836-839, Aug. 2005.

[4] S. Hong, B. Kim, and D. Eom, "A base-station centric data gathering routing protocol in WSNs useful in home automation applications", IEEE Trans. of Cons. Elec., vol. 53, no. 3, pp. 945-951, Aug. 2007.

[5] S. Zacharias, T. Newe, S. OKeeffe, and E. Lewis, "Identifying sources of interference in RSSI traces of a single IEEE 802.15.4 channel," in Proc. IARIA ICWMC, Venice, Italy, pp. 408-414, Jun. 2012.

[6] C. Gomez, and J. Paradells, "Wireless home automation networks: a survey of architectures and technologies," in IEEE Communications Magazine, vol. 48, no. 6, pp. 92101, Jun. 2010.

[7] A. J. D. Rathnayaka, V. M. Potdar, and S. J. Kuruppu, "Evaluation of wireless home automation technologies," in Proc. IEEE DEST, Daejeon, Korea, pp.76-81, Jun. 2011.

[8] ZigBee Alliance, ZigBee home automation public application profile. [Online]. Available: https://docs.zigbee.org/zigbee-docs/dcn/07/docs-07-536702-0afg-home-automation-profile-for-public-download.pdf, accessed Jul. 2015.

[9] C. E. Perkins, and E. M. Royer, "Ad hoc on-demand distance vector routing," in Proc. IEEE WMCSA, New Orleans, pp. 90-100, 1999.

[10] I. D. Chakeres, and K. B. Luke, "AODV ${ }_{\mathrm{jr}}$, AODV simplified," in Proc. ACM SIGCOMM Mob. Comp. and Comm. Rev., vol. 6, no. 3, pp. 100-101, Jul. 2002.

[11] M. Galeev, "Catching the Z-Wave," Embedded Systems Design J., vol. 19, no. 10, pp. 1-5, Oct. 2006.

[12] P. Darbee, "INSTEON: the details," Tech. Rep., v2, pp. $1-58,2013$.

[13] A. Garca-Hernando, J. Matnez-Ortega, J. Lpez-NaVArro, A. Prayati, and L. Redendo-Lpez, Problem solving for WSNs, 1st ed., Springer, pp. 136-137, Jul. 2008.

[14] X. Li, S. H. Hong, and K. Fang, "Location-based selfadaptive routing algorithm for WSNs in HA," EURASIP J. of Embed. Sys., 2011.

[15] X. H. Li, S. H. Hong, and K. L. Fang, "WSNHA-GAHR: a greedy and $A^{*}$ heuristic routing algorithm for WSNs in home automation," IET Comm., vol. 5, no. 13, pp.17971805, Sep. 2011. 
[16] K. Biswas, V. Muthukkumarasamy, E. Sithirasenan, and K. Singh, "An energy aware heurictic based routing protocol for WSNs," in Proc. IEEE ICCIT, Dhaka, pp. 53-58, Dec. 2014.

[17] A. Savvides, C. Han, and M. Srivastava, "Dynamic finegrained localization in ad-hoc networks of sensors," in Proc. ACM MobiCom., Rome, Italy, pp. 166-179, Jul. 2001.

[18] W. B. Heinzelman, A. P. Chandraprakasan, and H. Balakrishnan, "Application specific protocol architecture for wireless microsensor networks," IEEE Trans. on Wireless Net., vol. 1, no. 4, pp. 660-670, 2002.

[19] D. Tudose, L. Gheorghe, and N. Tapus, "Radio transceiver consumption modeling for multi-hop WSNs," in UPB Scientific Bulletin, Series C, vol. 75, no. 1, pp. 17-26, 2013.

[20] W. Kim, J. Lee, T. Kwon, S. Lee, and Y. Choi, "Quantifying the interference gray zone in wireless networks: a measurement study," in Proc. IEEE ICC, pp. 3758-3763, Jun. 2007.

[21] D. Son, B. Krishnamachari, and J. Heidemann, "Experimental study of concurrent transmission in WSNs," in Proc. ACM SenSys, New York, USA, pp. 237-250, 2006.

[22] M. Volker, B. Katz, and D. Wagner, "On the complexity of scheduling with power control in geometric SINR," Fakultat fur Informatik, Universitat Karlsruhe, Tech. Rep., pp. 1-10, Aug. 2009.

[23] W. Zeng, R. L. Church, "Finding shortest paths on real road networks: the case for $A^{*}$," Int. J. of Geographical Information Science, vol. 23, no. 4, pp. 531-543, 2009.

Kamanashis Biswas received his Masters in Security Engineering from Blekinge Institue of Technology, Sweden in 2007. He is currently a Ph.D. student in School of ICT, Griffith University, Gold Coast, Australia. His research interests include cryptography, IDS, energy efficient and secure routing, and clustering in WSNs.

Vallipuram Muthukkumarasamy obtained B.Sc. Eng. with 1st Class Hons. from University of Peradeniya, Sri Lanka and obtained Ph.D. from Cambridge University, England. $\mathrm{He}$ is currently attached to School of Information and Communications Technology, Griffith University, Australia an Associate Professor. His current research areas include investigation of security issues in wireless networks, sensor networks, trust management in MANETs, key establishment protocols and medical sensor networks. He is currently leading the Network Security research Group at the Institute for Integrated and Intelligent Systems at Griffith University. He has also received a number of best teacher awards.

Xin-Wen Wu received the Ph.D. degree from the Chinese Academy of Sciences. He was with the Chinese Academy of Sciences, the University of California, San Diego (as a post-doctoral researcher), and the University of Melbourne (as a research fellow). He was affiliated with the School of Information Technology and Mathematical Science, University of Ballarat, Australia. In April 2010 he joined Griffith University, Australia, as a faculty member of the School of ICT. His research interests include network and data security, coding techniques, and information theory and its applications. He has published one book, three book chapters and over sixty research papers in IEEE transactions, other journals, and conferences proceedings.

Kalvinder Singh is a researcher and developer for Australia Development Labs, IBM, in the security division. He received a Doctorate from Griffith University, Gold Coast, Queensland, Australia. His research interests include authentication protocols, cryptography, network security, intrusion detection, sensors and body sensors. 